\title{
STREPTOCOCCAL INTERMEDIUS BRAIN ABSCESSES IN AN ADOLESCENT MALE WITH HISTOPLASMA
} INFECTION

Michael E Watson Jr, S Celeste Morley

Keywords: Streptococcus intermedius, brain abscesses, Histoplasma

\begin{abstract}
Intracerebral abscesses are associated with significant morbidity and mortality. We report the case of a healthy 16 year-old male who presented with seizure and was found to have multifocal Streptococcus intermedius brain abscesses. Further studies determined the patient had necrotizing hilar lymphadenopathy and pneumonia by chest computed tomography (CT) and positive serologic testing for infection by Histoplasma capsulatum. The patient was treated with meropenem and recovered completely. The histoplasma infection resolved without anti-fungal therapy.
\end{abstract}

\section{Introduction}

Intracerebral abscesses due to bacterial infection are a medical emergency in children and adolescents as they are associated with potential for serious neurological sequelae or death. $(1,2)$ Contiguous spread of bacteria from infections in the oropharynx, middle ear and paranasal sinuses has traditionally been the source of most brain abscesses. (3) Hematogenous dissemination of bacterial pathogens causing brain abscesses can occur with predisposing conditions including pneumonia, bacterial endocarditis or dissemination from distal sites (e.g., abdominal abscess) when a right to left intracardiac shunt exists. (1) We present an illustrative case of an adolescent male with multifocal brain lesions due to Streptococcus intermedius along with Histoplasma capsulatum coinfection.

\section{Case Report}

A 16 years old male from rural Illinois presented with a generalized tonic-clonic seizure exceeding 20 minutes in duration for which he was intubated and mechanically ventilated. This episode was preceded by 10 days of fever, rhinorrhea, and mild cough, for which treatment with azithromycin for 5 days provided no improvement. He had no prior history of seizures and his past medical history was unremarkable. His immunizations were up to date with current recommendations. He had no significant exposures and no recent travel. On examination, the patient was well-grown ( 82.8 $\mathrm{kg}$, 90th percentile weight for age), drowsy with temperature of $101.50 F$. General and systemic examinations were normal. A complete blood count showed an elevated leukocyte count of 21,200 cells/cumm with differential count of $84 \%$ neutrophils, $8 \%$ lymphocytes, and 8\% monocytes. Head computerized tomography (CT) revealed multiple hypoattenuation lesions with hyperdense rims suggestive of abscesses. Lumbar puncture showed cloudy, colorless cerebrospinal fluid (CSF) with elevated closing pressure of $36 \mathrm{~cm}$ water though opening pressure was not documented. CSF cell count was 1500 cells per high power field, with differential of $49 \%$ neutrophils, $48 \%$ monocytes, and $3 \%$ lymphocytes. CSF protein was $326 \mathrm{mg} / \mathrm{dl}$ and glucose was $39 \mathrm{mg} / \mathrm{dl}$ (serum glucose was $134 \mathrm{mg} / \mathrm{dl}$ ). A brain magnetic resonance imaging (MRI) showed 13 individual brain abscess lesions consistent with septic emboli (Fig. 1). Trans-thoracic and trans-esophageal echocardiograms did not show endocarditis or structural defects. Chest, abdomen, and pelvis CT scan showed extensive necrotic hilar and subcarinal lymphadenopathy with calcifications, along with pneumonia of the bilateral lower lung fields (Fig. 2). The radiologist's interpretation was that the lymphadenopathy was more consistent with an underlying fungal infection given the necrosis and calcifications, and less likely to be reactive adenopathy from pneumonia.

Patient was treated initially with vancomycin and meropenem. CSF aerobic, fungal, tuberculosis (TB) cultures showed no growth. Polymerase chain reaction (PCR) of CSF for Herpes Simplex Virus (HSV), Epstein Barr Virus (EBV), and Toxoplasma were negative. CSF Cryptococcal antigen testing was negative. Blood aerobic, anaerobic, and fungal cultures had no growth. Biopsy of a superficial abscess lesion located in the right temporal bone area revealed Gram-positive cocci in pairs and chains and culture grew abundant Streptococcus intermedius. Anaerobic, fungal, and TB cultures of the brain abscess fluid showed no growth. Meropenem was continued for coverage of the S. intermedius and for the possibility of additional anaerobic organisms not recovered in culture. We can
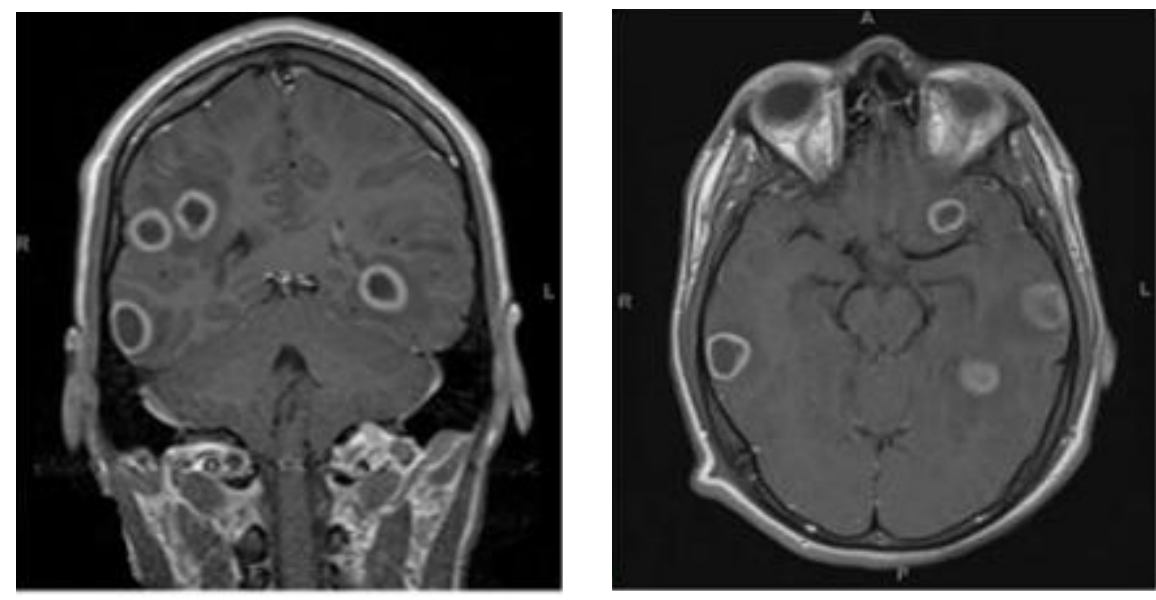

Figure 1. Coronal and transverse MRI T1 images of the brain demonstrating multiple ring-enhancing lesions of the supratentorial brain consistent with septic emboli. Additional images revealed a total of 13 intracerebral abscess lesions in the patient. 

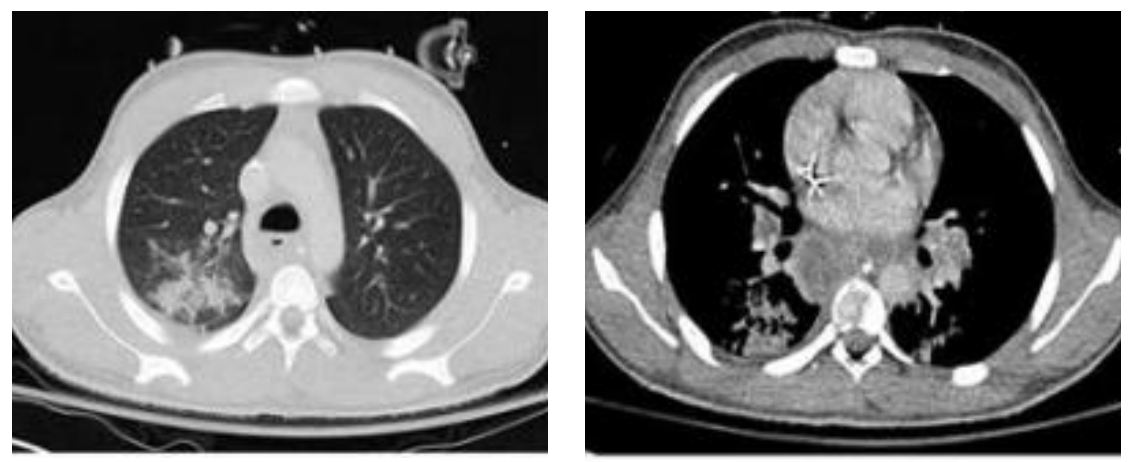

members of the Enterobacteriaceae. Immune compromised individuals with brain abscesses are often infected with more exotic pathogens such as Cryptococcus, Histoplasma, Nocardia, and Toxoplasma. $(1,7)$ Of the three species within the $\mathrm{S}$. anginosus group, $\mathrm{S}$. intermedius has been shown to have the greatest association with abscesses within the central nervous system (CNS). Most patients with CNS abscesses due to $\mathrm{S}$. intermedius are immunocompetent, although

Figure 2. Transverse computed tomography (CT) images of the chest demonstrating extensive hilar and subcarinal lymphadenopathy with necrosis and calcifications. Airspace opacities within the right upper lobe, right lower lobe, and left lower lobe, are consistent with multifocal pneumonia.

predisposing factors including cardiac and pulmonary anomalies (right to left shunting lesions), sinusitis, frequent otitis media, and dental disease, may serve as

only speculate that the source of $\mathrm{S}$. intermedius was dissemination from a pulmonary source, possibly the pneumonia, or superinfection of a necrotizing hilar lymph node, a reported but uncommon complication of infection with histoplasmosis or tuberculosis. (4) Serology for Histoplasma was positive with yeast phase complement fixation titers of $1: 32$ (normal $<1: 8$ ). Immunodiffusion and urine Histoplasma antigen were negative, as were serologies for Blastomyces and Coccidioides. Since he was not symptomatic prior to this acute illness and given the high prevalence of subclinical infection with Histoplasma capsulatum in the Midwest United States, he was diagnosed with pulmonary histoplasmosis, but no specific treatment was initiated as it is not usually required for resolution in immunocompetent individuals. (5) An evaluation for possible immunodeficiency including HIV serology, immunoglobulin class and subclass levels, lymphocyte subpopulation counts, complement components, and neutrophil oxidative burst assay were normal.

In follow-up, he was treated with 6 weeks of meropenem followed by two weeks of oral amoxicillin-clavulanate. Repeat brain MRI demonstrated improvement in the appearance of the abscess lesions with eventual resolution. Repeat serology for Histoplasma two months later again showed reduction in yeast phase complement fixation titers to $1: 16$.

\section{Discussion}

Streptococcus intermedius is a member of the Streptococcus anginosus group, which consists of 3 species ( $\mathrm{S}$. intermedius, $\mathrm{S}$. anginosus, and $\mathrm{S}$. constellatus). The $\mathrm{S}$. anginosus group members are commensals and can be isolated from the oropharynx, sinuses, gastrointestinal, and genitourinary tracts, but can be associated with disease including a propensity for abscess formation. (6)

Common pathogens from brain abscesses include members of the Streptococcus anginosus group, Staphylococcus aureus, Bacteroides species, and access points for bacterial penetration of the CNS. (8) Culture of biopsy material or blood is considered the gold-standard for diagnosis, although reports of using $16 \mathrm{~S}$ rRNA amplification and sequencing in culturenegative cases have proved beneficial in identifying $S$. anginosus group infections. $(9,10)$ Empiric antibiotic therapy should include coverage for Gram-positive and Gram-negative aerobic and anaerobic organisms, typically including vancomycin and a carbapenem (or combined metronidazole plus 3rd or 4th generation cephalosporin). (1)

Infection with Histoplasma capsulatum variety capsulatum causes symptoms in less than $5 \%$ of those infected. Acute pulmonary histoplasmosis may present as an influenza-like illness with nonpleuritic chest pain, hilar adenopathy, and pulmonary infiltrates, lasting up to 2 weeks. (11) The disease is endemic in certain areas of the United States, particularly within the Ohio, Missouri, and Mississippi River valleys. The organism grows in moist soil and spores are inhaled into the lungs. Treatment is not routinely recommended for immunocompetent children with pulmonary histoplasmosis, but may be indicated in severe infections with infants, immunocompromised children, or in cases where granulomatous adenitis obstructs local bronchi or blood vessels. In these serious cases, amphotericin $B$ and/or itraconazole may be required for duration of several weeks to months $(5,11)$.

Intracerebral abscesses represent an uncommon infectious disease emergency requiring an expedited and multidisciplinary approach for diagnosis and management. An extensive search for underlying risk factors or sources of dissemination is appropriate for optimal treatment of these infections.

Funding: Michael Watson is supported by a Pediatric Infectious Diseases Society Fellowship Award generously funded by GlaxoSmithKline.

Author Contributions: MEW and SCM both contributed to the preparation of this manuscript. 


\section{References}

1. Honda $\mathrm{H}$, Warren DK. Central nervous system infections: meningitis and brain abscess. Infect Dis Clin North Am 2009; 23: 609-623

2. Mathisen GE, Johnson JP. Brain abscess. Clin Infect Dis 1997; 25: 763-779

3. Mamelak AN, Mampalam TJ, Obana WG, Rosenblum ML. Improved management of multiple brain abscesses: a combined surgical and medical approach. Neurosurgery 1995; 36: 76-85

4. Schneider RD, Reid JD. Mediastinal histoplasmosis with abscess. Chest. 1975; 67: 237-239

5. Wheat LJ, Freifeld AG, Kleiman MB, Baddley JW, McKinsey $D S$, Loyd JE, et al. Clinical practice guidelines for the management of patients with histoplasmosis: 2007 update by the Infectious Diseases Society of America. Clin Infect Dis 2007; 45: 807-825

6. Gray T. Streptococcus anginosus group: clinical signficance of an important group of pathogens. Clin Microbiol Newsletter 2005; 27: 155-159

7. Lewin JL, Lapointe M, Ziai W. Central nervous system infections in the critically ill. J Pharm Pract. 2005; 18: 25-41

8. Whiley RA, Beighton D, Winstanley TG, Fraser HY, Hardie JM. Streptococcus intermedius, Streptococcus constellatus, and Streptococcus anginosus (the Streptococcus milleri group): association with different body sites and clinical infections. J Clin Microbiol. 1992; 30: 243-244

9. Petti CA, Simmon KE, Bender J, Blaschke A, Webster $K A$, Conneely MF, et al. Culture-Negative intracerebral abscesses in children and adolescents from Streptococcus anginosus group infection: a case series. Clin Infect Dis 2008; 46: 1578-1580.

10. Claridge JE, 3rd, Attorri S, Musher DM, Hebert J, Dunbar S. Streptococcus intermedius, Streptococcus constellatus, and Streptococcus anginosus ("Streptococcus milleri group") are of different clinical importance and are not equally associated with abscess. Clin Infect Dis. 2001; 32: 1511-1515.

11. Fischer GB, Mocelin H, Severo CB, Oliveira Fde M, Xavier MO, Severo LC. Histoplasmosis in children. Paediatr Respir Rev 2009; 10: 172-177

From: Division of Pediatric Infectious Diseases, Department of Pediatrics, Washington University School of Medicine, St. Louis, Missouri, USA.

Address for Correspondence: Dr Michael E. Watson Jr, Washington University School of Medicine, Campus Box 8230, 660 S. Euclid Ave, St. Louis, MO, 63110, USA. Email: watson_m@kids.wustl.edu.

E-published: 1st October 2012 . Art\#62

DOI No. 10.7199/ped.oncall.2012.62

Quick Response Code

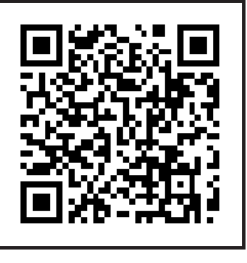

